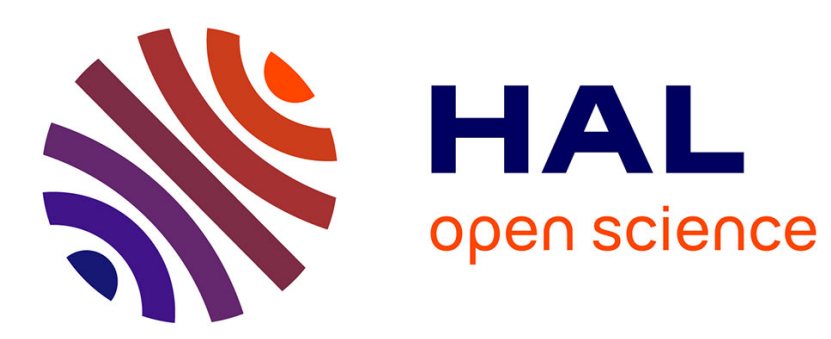

\title{
A meta-analysis of the effectiveness of the low-ball compliance-gaining procedure
}

\author{
A. Pascual, C.J. Carpenter, N. Guéguen, F. Girandola
}

\section{To cite this version:}

A. Pascual, C.J. Carpenter, N. Guéguen, F. Girandola. A meta-analysis of the effectiveness of the low-ball compliance-gaining procedure. European Review of Applied Psychology / Revue Européenne de Psychologie Appliquée, 2016, 66 (5), pp.261-267. 10.1016/j.erap.2016.06.004 hal-03175652

\section{HAL Id: hal-03175652 \\ https://hal.science/hal-03175652}

Submitted on 9 Jan 2022

HAL is a multi-disciplinary open access archive for the deposit and dissemination of scientific research documents, whether they are published or not. The documents may come from teaching and research institutions in France or abroad, or from public or private research centers.
L'archive ouverte pluridisciplinaire HAL, est destinée au dépôt et à la diffusion de documents scientifiques de niveau recherche, publiés ou non, émanant des établissements d'enseignement et de recherche français ou étrangers, des laboratoires publics ou privés. 


\section{Personality and Social Psychology Review}

\section{A Meta-Analysis of the Effectiveness of the Low-Ball Compliance Gaining Procedure}
A. Pascual, University of Bordeaux
CJ Carpenter Western Illinois University,
N. Guéguen University South Brittany
F. Girandola Aix-Marseille University

\begin{tabular}{|r|l|}
\hline Journal: & Personality and Social Psychology Review \\
\hline Manuscript ID: & PSPR-02-15-014 \\
\hline Keywords: & $\begin{array}{l}\text { Applied Social Psychology, Helping/Prosocial Behavior, Social } \\
\text { Influence/Power }\end{array}$ \\
\hline
\end{tabular}


1

2

3

4

5

6

7

8

9

10

11

12

13

14

15

16

17

18

19

20

21

22

23

24

25

26

27

28

29

30

31

32

33

34

35

36

37

38

39

40

41

42

43

44

45

46

47

48

49

50

51

52

53

54

55

56

57

58

59

60

A Meta-Analysis of the Effectiveness of the Low-Ball Compliance Gaining Procedure 


\begin{abstract}
The low-ball technique (Cialdini et al., 1978) is a compliance gaining technique consisting in making a very attractive initial offer to get a person to agree to the request and then making the terms less favorable. The effectiveness of this technique was evaluated in a meta-analysis using 17 references, including 23 studies, 45 subgroups and a combined sample size of 5218 . The completed analysis reported a weighted mean correlation coefficient of $r=.16$ and a weighted mean odds ratio of $\mathrm{OR}=2.47$. Moderator analysis reported that the Low-ball technique was more efficient with a high cost for the participant and when the solicitation of the target request is deferred. These findings appeared congruent with commitment theoretical explanation of the Low-Ball technique.
\end{abstract}

Keywords: Low-ball technique, Request, Compliance, Meta-analysis 
Many techniques has been found in social psychological literature for gaining compliance with a request: the foot-in-the-door technique (Freedman \& Fraser, 1966), the doorin-the-face technique (Cialdini, Vincent, Lewis, Catalan, Wheeler, \& Darby, 1975), the foot-inthe-mouth (Howard, 1990), the "evoking freedom" technique (Guéguen et al., 2013), etc. (see Pratkanis, 2007 for an exhaustive review).

One of these procedures, the low-ball, appears to be very effective at increasing compliance with a request. However, unlike most of the other techniques cited, it has received less interest from researchers. The low-ball technique (Cialdini, Cacioppo, Bassett \& Miller, 1978 ) is based on a simple principle: obtaining an individual's agreement to engage in a behavior either by painting the advantages in glowing colors which disappear afterwards, or by hiding the drawbacks which arise after the initial acceptance. The individual is then asked again if he/she still agrees to do what had been asked of him/her.

\section{The low-ball technique}

In the first experimental study carried out on the low-ball technique, Cialdini, Cacioppo, Bassett and Miller (1978) hoped that students would agree to participate in a psychological experiment which was scheduled very early in the morning. The experimenter recruited students in classrooms by saying that he was looking for students who would be given a course credit for participating in a study on cognitive processes. The experimenter was honest in the control situation and said that the experiment would begin at 7 am. In the lowball condition, the experimenter asked if there were volunteers to participate in a psychology experiment. After one participant accepted, the experimenter informed him that the experiment would be held at $7 \mathrm{am}$, and then he asked the participant if he was still willingto participate in the experiment. The participant was given the date and place of the appointment for the experiment in both conditions. The day before the appointment, each participant was contacted by phone to be reminded. The results show that $31 \%$ of the 
participants in the control condition accepted the final request compared to $56 \%$ in the experimental condition. Moreover, the behavioral measurements show that $24 \%$ of the participants in the control group arrived at the fixed time compared to $53 \%$ in the low-ball condition.

According to these authors, the low-ball effect can be explained by the theory of commitment (Kiesler, 1971). This theory supports the hypothesis of a psychological mechanism of resistance to change. It seems that once the decision to help the solicitor is made, the participant sticks to that decision even if the cost of the help has significantly increased. The individual who is committed to the behavior will then attempt to act in accordance with his/her first decision even if the new behavior is less rewarding.

The effectiveness of this technique has been shown in numerous studies. It was reported that the low-ball technique increases compliance with a pro-social request (Burger \& Cornelius, 2003; Guéguen, Pascual, \& Dagot, 2002; Weyant, 1996). Research also found that this technique appeared effective to encourage smokers to stop smoking during 18 hours (Joule, 1987) or to stop eating during 12 hours (Joule, Fointiat, Pasquier \& Mugny, 1991). It was also found that the low-ball increased user contributions to online communities (Masli \& Terveen, 2012).

The objective of this paper was triple. First, we wanted to evaluate the mean of effectsize of this compliance technique given that some research reported a positive effect of the low-ball technique relative to the control group (e.g. Joule, 1987) and others reported a reverse effect with the low ball technique decreasing compliance with the request (e.g. Motes \& Woodside, 1979). Second, we wanted to compare the mean low-ball effect-size with further well-known compliance techniques for which several meta-analysis were performed: the footin-the-door (Beaman, Cole, Preston, Klentz, Merhrkens \& Steblay, 1983; Dillard, Hunter \& Burgoon, 1984; Fern, Monroe \& Avila, 1986; Burger, 1999; Pascual \& Guéguen, 2005), the 
Door-in-the-Face (Dillard, Hunter \& Burgoon, 1984; Feeley, Anker \& Aloe, 2012; O’Keefe \& Hale, 2001), the "but you are free to..." technique (Carpenter, 2013), the Legitimization of Paltry Contribution technique (Andrews et al., 2008) or the Disrupt-the-reframe technique (Carpenter \& Boster, 2009). As most of the requests used with the compliance techniques are nearly the same as those used in low-ball literature, the comparison of the effect-sizes reported with the different techniques are possible. Third, we wanted to test the effect of several possible moderators of the effectiveness of low-ball technique. Some studies on lowball techniques have used different types of requests. For some there was a delay or none between the first and the second request. Additionally the request has been stated face-to-face, by telephone, or by internet. Thus, it could be interesting to test the effect of such possible mediators on compliance with a request because several of the meta-analyses performed on well-known compliance-gaining procedures have reported have found evidence that these variables moderate compliance-gaining effectiveness.

\section{Method}

\section{Sample}

Several disciplinary search engines were used to find articles: PsyInfo, Ebsco Business Source Premier, Communication and Mass Media Complete, Science Direct, MedLine. General Search engines were also used (Web of Science, HAL, Google Scholar). A more general Web search completed the process. The terms "low-ball" and "low ball" were used as search terms in all the cases.

\section{Study selection}

Each study using the low-ball procedure was considered for inclusion in the metaanalysis. However, only studies that had a control group with only the later request addressed to the participants and a Low-ball group with the two classical requests were included. This criterion excluded 3 studies (Burger \& Cornelius, 2003, study 1; Burger \& Petty, 1981, study 
2 and 3; Wang, Brownstein, \& Katzev, 1989). Only studies that had a dichotomous complied/did not complied dependent variable were retained. This excluded some continuous dependent variables that appeared in some studies (Browstein \& Katzev, 1985; Hornik, Zaig, \& Shadmon, 1991a; Masli \& Terveen, 2012). However, in these later studies the first dependent variable was a dichotomous complied/did not complied one. Thus, these studies were included in the meta-analysis. Furthermore, two references were excluded from analysis (Hornik, Zaig, Shadmon, \& Barbash, 1990; Hornik, Zaig, \& Shadmon, 1991b) because their data were published in another reference (Hornik, Zaig, \& Shadmon, 1991a). At the end, we found a set of 17 articles reporting the results of 23 studies including a total of 4885 participants. Table 1 presents the rate of compliance of both conditions and sample-sizes of each study used in the meta-analysis.

- Table 1 Here -

\section{Coding}

Several factors associated with each study were coded as possible moderators in this paper. Nine moderators were considered in the meta-analysis:

- type of request (prosocial, self-interested...),

- cost for the participants (low, moderate or high),

- the country where the study was performed,

- the delay between the first and the second request in the low-ball condition (immediate or with a delay),

- whether the request was addressed to a group of participants or to a single participant.

- the presentation mode of the request (face-to-face, telephone...),

- whether the study measured verbal compliance with the request or behavioral compliance,

- when the information was available, we coded the gender of the participants, 
- also in cases when the information was available the gender of the solicitor was coded.

After decomposing the studies by moderator, 45 sub-groups were found.

For all the experimental cases included in meta-analysis, two effect-sizes were coded. First, according to Cooper (1998) we calculated the correlation coefficient resulting from the comparison between the Low-Ball technique compared with the control direct-request situation. We also calculated the odds ratio (OR) for each experimental case. Of course, OR was calculated only when the compliance measure was a dichotomous (comply or not with the request) one. This excluded the second dependent variables measured in Browstein and Katzev (1985), Hornik et al., (1991a); Masli and Terveen (2012).

\section{Results}

Initially, the various meta-analytic statistics that were calculated and their interpretation will be described. Then the overall results will be reported. Then the effects of the coded moderators of the effect will be explored.

Table 2 displays the information about the overall findings and the subgroups analysis. The first column, "k," indicates how many studies were included in that calculation. The "N" column indicates how many total subjects participated in those studies. The "r" column indicates the effect of using the low-ball technique relative to a direct request control expressed as a correlation such that positive correlations indicate the low-ball was more effective. These are sample size weighted estimates of the average effect as per Hunter and Schmidt's (2004) recommendations. The "OR" column represents the odds ratio such that a value greater than one indicates that the subjects more likely to comply in the low-ball condition than the control. For example an OR of two indicates the low-ball subjects were twice as likely to comply. The ORs were calculated and averaged using recommendations from Haddock, Rindskopf, and Shadish (1998).

\section{- Table 2 Here -}


The remaining three columns indicate the extent to which the specified sample of studies represents a homogeneous effect and are again based on Hunter and Schmidt's (2004) recommendations. A homogeneous effect is one that estimates a true, single population value for that group. A homogeneous effect is unmoderated such that all the variation in the effect size is only due to sampling error. These effects are also called fixed effects. A heterogeneous effect estimate does not represent a single population effect (also called a random effect). Instead, it represents the average of several population effects. These several effects are those that are based on moderators. For example, in this meta-analysis, the overall effect is heterogeneous and thus is moderated. There is not a single population effect for the low ball because the effect depends on a number of additional conditions. Yet, when the studies that used prosocial requests are considered as a group, that effect is homogeneous and can be taken as an estimate of the effectiveness of the low-ball technique when the request is prosocial. The "\% explained" column represents Hunter and Schmidt's method of estimating if the effect is homogeneous. That column indicates how much of the variation in effect sizes is due to sampling error. They argue that if about $75 \%$ or more of the variation is due to sampling error, the effect is likely to be homogeneous, i.e. unmoderated.

The next two columns represent the $80 \%$ credibility interval. A credibility interval is different than a confidence interval and in the context of a meta-analysis is more useful whereas the confidence interval is biased (Hunter \& Schmidt, 2004). The credibility interval indicates the expected variation in the effect size due to additional moderators. For example, the full sample effect size is expected to vary between -.04 and .38 based on moderators. In other words, some moderators will cause the effect to be null and some will cause it to be stronger. When the "\% explained" column indicates 100, it means that there is not any variation due to moderators so a credibility interval cannot be constructed.

\section{Main Findings}


The first row of data in Table 2 shows the results of the overall meta-analysis containing every obtained study. The overall correlation was somewhat small $(r=.16)$ but consistent with other compliance-gaining techniques. The $27 \%$ variance explained suggests that the effectiveness of the technique was moderated.

As the Hunter and Schmidt (2004) method weights the average effect size by sample, it is sensitive to outliers in the sizes of the samples in the study. One study, the only study conducted via the internet, had an unusually large sample $(N=1,904)$ which represented $36 \%$ of the total combined sample. That study found an unusually small effect $(r=.03)$. Given the combination of an unusually small effect, a large sample size, and a unique message channel, it may be more useful to consider the overall effect of the technique in face-to-face or telephone delivery. When that study was removed from the data set, the overall effect was larger $(r=.23)$ and the explained variation was higher $(37 \%)$. The second row of data represents the results of studies conducted using the traditional channels of face-to-face and telephone. Given this potential outlier, all moderator analyses are displayed both with and without the internet study in the results table. It is our position that the moderator analysis shown without the internet study (rows with "w/o Int") present more accurate results, but both are included.

\section{Moderator Analysis}

The first moderator to be considered was the nature of the request. There were three types of requests found in the literature: business, self-interested, and prosocial. Requests not fitting into these three were placed in the "other" category. As Table 2 shows, the business requests found the weakest effect for the technique, the prosocial a moderate effect, and the selfinterested the largest. In the prosocial request studies, the set was homogeneous and unlikely to be further moderated whereas the other three groups were not. The credibility interval for the business group included a nearly null effect which suggests there are times 
when the technique will produce almost no effect for a business request. The credibility interval for the self-interested requests, on the other hand, suggests that the technique will almost always be moderately effective for that type of request.

Next the cost to the target will be considered. The data indicate that when using the low-ball for low to moderately sized requests the technique will have a somewhat small effect. The credibility interval suggests that there are occasions when the technique will barely work at all for these requests. Yet when the request has a high cost for the target, the technique reaches a moderately sized effect that is unlikely to disappear regardless of the moderator.

There were two primary countries in which most of the studies took place: France and the United States. Two were conducted in Poland and two in Israel, but two is an insufficient number for meta-analysis. The technique tended to be substantially larger in France than in the United States. Without the internet study, the credibility interval indicates that the effect in the U.S. was moderate but may disappear in some situations whereas the effect in France may be small but will tend to produce a noticeable effect regardless of the moderator.

Closer examination of the set of French and set of American studies showed that all of the self-interested request studies were French whereas there were no French business request studies. This pattern raised the possibility than the increase in effectiveness associated with French studies may be attributed to those studies more frequently using the more effective self-interested requests. The lower portions of the Table show the country findings broken down further by the type of request. There were business request studies in the U.S. set but none in France and there were self-interested request studies in France but not in the U.S. Yet, there were prosocial request studies conducted in both countries as well as those placed in the "other" category. The prosocial studies in France showed a somewhat larger effect than the U.S. and both of these effects were homogeneous. That suggests that there are unlikely to be 
any additional moderators that explain this difference. That finding indicates that even when the type of request is the same, the French studies tend to find a larger effect. Additional research is needed on business requests in France and self-interested requests in the U.S. in order to determine if the increased effectiveness associated with French samples occurs with those types of requests as well.

Some of the studies required a response immediately whereas in others it was deferred. The results suggest that the technique is stronger when the target request is deferred. Without the internet study, the effect is somewhat larger for the deferred than the immediate, but there is a large overlap in credibility intervals which indicates that there are many occasions in which the effect will be the same size. The difference does suggest a somewhat larger tendency for people to comply in the deferred decision studies.

The next moderator was whether the target of the request was an individual or a group. There were only three studies for which the target was a group so results should be considered very tentative. Despite the small group, the set was homogeneous and the effect was very small. The technique may not be effective when the target is a group rather than an individual.

Another moderator was whether the request was made face to face, over the phone, or via the internet. The small effect size associated with the single internet study has already been mentioned. The phone studies seem to find a somewhat larger effect than the face to face studies. There is some overlap in the credibility intervals that suggests that although the phone will usually be more effective, there are some situations in which a face to face request may be as effective as the phone.

The studies also varied depending on whether or not the measure was verbal agreement to perform a behaviour or if the behaviour was actually observed. When the internet study was excluded from the behavioural set, there was very little difference between 
the two measures. Both were heterogeneous. This proposed moderator variable seems to have had little effect on the low-ball's effectiveness.

Next, the sex of the experimenter was examined. In many studies, both male and female experimenters were used $(\mathrm{k}=25)$ but in some only female or only male were used. The results indicate that the technique was more effective when female experimenters were used than male. These estimates were homogeneous, which suggest that female experimenters were consistently more effective than male in these studies. Oddly, studies that used both, when the internet study is excluded, find an effect that is nearly as large as those using exclusively females. These results may indicate that using only male requesters is an less effective means of implementing the technique.

Additionally, some studies exclusively targeted women or exclusively men, though most targeted both. In this case, male targets tend to be more compliant than female. The female target sample can be considered homogeneous as more than $75 \%$ of the variance was explained by sampling error. The male target sample was not far off from the threshold for homogeneity, suggesting that a small amount of variance could be accounted for by additional moderators. Without the internet study, the studies that targeted both sexes produced a heterogeneous effect that fell between the male and female effects. Closer examination of the exclusively male and exclusively female target groups found that these studies were also consistently face to face whereas the studies that targeted both sexes included face to face and telephone studies. When only the studies that targeted both sexes and used a face to face interaction were examined as a set, the effect size was almost identical to the female subjects' effect. It appears that if the low-ball is implemented face to face, targeting men is more effective.

\section{Publication Bias}


In some research literatures, studies finding null effects are not published due to a bias against results that do not find statistically significant results. There are various methods of attempting to determine if a meta-analysis is missing studies that were not published due to their findings. One such method is the trim-and-fill algorithm (Duval \& Tweedie, 2000). The algorithm assumes that study effect sizes should have a predictable distribution based on sampling error when there are no missing studies. The current set of studies was tested using the trim-and-fill and the algorithm did not detect any missing studies in the data set. It appears that publication bias is unlikely to be a problem in this particular research area.

\section{Discussion}

The weighted mean effect size of the increased probability of compliance associated with the Low-Ball technique appeared similar to the meta-analytic estimates of the effect size reported with further compliance-gaining procedures such as the Foot-in-the-Door $(\mathrm{r} \approx .10$ : Beaman, Cole, Preston, Klentz, Merhrkens \& Steblay, 1983; Dillard, Hunter \& Burgoon, 1984; Fern, Monroe \& Avila, 1986; Burger, 1999; Pascual \& Guéguen, 2005), the Door-inthe-Face ( $\mathrm{r} \approx .13$, Dillard, Hunter \& Burgoon, 1984; Feeley, Anker \& Aloe, 2012), the "but you are free to..." technique $(r=.13$ : Carpenter, 2013) or the Legitimization of Paltry Contribution technique $(r=.11$ : Andrews et al. (2008). Though the effect-size of the lowball appeared moderate $(r=.16)$, when excluding the one study conducted via the internet we reported a higher effect-size suggesting that the low-ball technique is an efficient way to increase compliance with a request

The moderator analysis reported that the type of the request was a significant factor with self-interested having the largest effect-size. However, we also reported that the effect of the low-ball technique was higher in France that in US. This suggested a possible confound effect between type of request and country because in the studies conducted in France most of them used self-interested requests. Thus cultural effect could explain both the effects of the 
type of the request and the country difference. Several studies reported cultural differences in the efficiency of compliance gaining-procedures according to culture. Pascual et al. (2012) observed that the "but you are free of..." technique - a verbal compliance procedure that solicits someone to comply with a request by simply telling a person that he or she is free to accept or refuse the request- was more effective in individualistic cultures than in collectivist cultures. Kilbourne (1989) found that the well-known "foot-in-the-door" varied in its effectiveness across three different cultures (Netherlands, France and Germany). In the same way, Pascual and Guéguen (2004) reported a difference in the efficiency of the "foot-in-thedoor with implicit demand" when comparing the U. S. and France. However, with the lowball technique the studies examined in France and US was not the same. Thus, it will be interesting to conduct cross-cultural studies using the same request in the future in order to more precisely examine the effects of culture.

We reported that the effect of the low-ball technique was stronger when the target request response was deferred. According to Cialdini et al. (1978), the effectiveness of the low-ball strategy could be explained by the theory of commitment (Kiesler, 1971). This theory is based on the hypothesis of a psychological mechanism of resistance to change: once the decision to agree to a solicitation is made, people stick to that decision even if the cost of agreeing has significantly increased. Individuals who are committed to produce a behavior will attempt to act in accordance with their initial decision, even if the behavior is less rewarding than originally expected. Accordingly, it could be state than when a delay occurred between the first and the second request, then the participant has more time to feel more committed with his/her initial decision and then the probability of saying "no" to the second request decreases. Thus, in future studies it could be interesting to experimentally vary delays between the initial request and the second less favorable request. 
At least, we reported that the cost of the request exerted a moderating effect on the lowball technique with higher compliance found in studies in which there were high cost requests. This overall result was congruent with a recent study performed by Guéguen and Pascual (2014, study 2). These authors varied the cost of the request in their study. They asked participants to have their photograph taken for a local magazine, holding different objects: a bottle of mint syrup (non-problematic product), a bottle of beer (problematic request), or a bottle of absinthe (an illegal product). The author reported that the difference in compliance between the control group and the low-ball group increased as soon as the cost of the request increased. The authors stated that the participant were motivated to try and maintain the positive impression made on a stranger, even if the request appeared more problematic at the end. Thus, as they said "yes" to the requester during the first stage, the participants said "yes" to the second request in order to maintain the good first impression of themselves as helpers that they had generated initially.

In conclusion, this meta-analysis on the Low-Ball technique demonstrated that the overall efficiency of this technique for gaining compliance with a request was slightly higher than the overall effectiveness of other well-known compliance-gaining techniques such as the Foot-in-the-Door or the Door-in-the-Face. However, it was found that this technique appeared more effective for gaining compliance with requests with a high cost to the participant. It was also reported that the efficiency of the request increases when the solicitation of the target request is deferred. Both findings suggest that the commitment theory could be an interesting approach to evaluate the process that could explain the effect of the low-ball technique. Our results also suggested that possible cultural factors could influence the efficiency of this technique and that future research should replicate the same study in different countries. 


\section{References}

*References marked with an asterisk indicate studies included in one or more of the metaanalyses.

Andrews, K. R., Carpenter, C. J., Shaw, A. S., \& Boster, F. J. (2008). The legitimization of paltry favors effect: A review and meta-analysis. Communication Reports, 21, 59-69.

Beaman, A.L., Maureen Cole, C., Preston, M., Klentz, B. \& Steblay, N.M. (1983). Fifteen years of foot-in-the-door research, a meta-analysis. Personality and Social Psychology Bulletin, 9(2), 181-196.

*Beauvois, J.L., Joule, R.V. \& Brunetti, F. (1993). Cognitive rationalization and act rationalization in an escalation of commitment. Basic and Applied Social Psychology, $14,1-17$.

*Brownstein, R.J. \& Katzev, R.D. (1985). The relative effectiveness of three compliance techniques in eliciting donations to a cultural organization. Journal of Applied Social Psychology, 15, 564-574.

Burger, J.M. (1999). The foot-in-the-door compliance procedure: A multiple process analysis and review. Personality and Social Psychology review, 3, 303-325.

*Burger, J. M. \& Cornelius, T. (2003). Raising the price of agreement: Public commitment and the low-ball compliance procedure. Journal of Applied Social Psychology, 33, 923934.

*Burger, J. M., \& Petty, R. E. (1981). The low-ball compliance technique: Task or person commitment? Journal of Personality and Social Psychology, 40, 492-500.

*Burns, A.C. \& De Vere, S.P. (1982). An investigation of compliance-gaining techniques applied to a gasoline conservation device. Journal of Public Policy and Marketing, 1, 43-55. 
Carpenter, C.J. (2013). A meta-analysis of the effectiveness of the "but you are free" compliance-gaining technique. Communication Studies, 64, 6-17.

Carpenter, C.J. \& Boster, F.J. (2009). A meta-analysis of the effectiveness of the disrupt-thenreframe compliance gaining technique. Communication Reports, 22, 55-62.

*Cialdini, R. B., Cacioppo, J. T., Basset, R. \& Miller, J. A. (1978). Low-ball procedure for producing compliance: Commitment then cost. Journal of Personality and Social Psychology, 36, 463-476.

Cialdini, R.B., Vincent, J.E., Lewis, S.K., Catalan, J., Wheeler, D. \& Darby, B.L. (1975). Reciprocal concessions procedure for inducing compliance: the door-in-the-face technique. Journal of Personality and Social Psychology, 31, 206-215.

Cooper, H. (1998). Integrating Research: A Guide for Literature Reviews (2nd Ed.). Thousand Oaks, CA: Sage.

Dillard, J.P., Hunter, J. \& Burgoon, M. (1984). Sequential-request persuasive strategies metaanalysis of foot-in-the-door and door-in-the-face. Human Communication Research, 10, $461-488$.

*Dufourcq-Brana, M. (2007). L'influence d'une déclaration de liberté sur l'efficacité du pieddans-la-porte et de l'amorçage [The influence of a declaration of freedom for the efficiency of the foot-in-the-door and the low ball] unpublished doctoral dissertation, Université de BretagneSud, Lorient, France.

Duval, S., \& Tweedie, R. (2000). A nonparametric “trim and fill”' method of assessing publication bias in meta-analysis. Journal of the American Statistical Association, 95, 89-98.

Feeley, T. H., Anker, A. E. \& Aloe, A. M. (2012). The Door-in-the-Face persuasive message strategy: The first 35 years. Communication Monographs, 79(3), 316-343. 
Fern, E., Monroe, K. \& Avila, R. (1986). Effectiveness of multiple request strategies: A synthesis of research results. Journal of Marketing Research, 23, 144-152.

Freedman, J.L. \& Fraser, S.C. (1966). Compliance without pressure: The foot-in-the-door technique. Journal of Personality and Social Psychology, 4, 195-202.

Guéguen, N., Joule, R.V., Halimi-Falkowicz, S., Pascual, A., Fischer-Lokou, J. \& DufourcqBrana, M. (2013). I'm Free but I'll Comply With Your Request : Generalization and Multidimensional Effects of the "Evoking Freedom" Technique. Journal of Applied Social Psychology, 43, 116-137.

*Guéguen, N, \& Pascual, A. (2014). Low-ball and compliance: Commitment even if the request is a deviant one. Social Influence, 9, 162-171.

*Guéguen, N., Pascual, A. \& Dagot, L. (2002). Low-ball and compliance to a request: An application in a field setting. Psychological Reports, 91, 81-84.

Haddock, C. K., Rindskopf, D., \& Shadish, W. R. (1998). Using odds ratios as effect sizes for meta-analysis of dichotomous data: A primer on methods and issues. Psychological Methods, 3, 339-353.

Hunter, J. E., \& Schmidt, F. L. (2004). Methods of meta-analysis: Correcting for error and bias in research findings. Thousand Oaks, CA: Sage.

*Hornik, J., Zaig, T. \& Shadmon, D. (1991a). Increasing compliance in costly telephone interviews: A test of four inducement techniques. International Journal of Research in Marketing, 8, 147-153.

Hornik, J., Zaig, T. \& Shadmon, D. (1991b). Reducing refusals in telephone surveys on sensitive topics. Journal of Advertising Research, 31, 49-56.

Hornik, J., Zaig, T., Shadmon, D. \& Barbash, G. I. (1990). Comparison of three inducement techniques to improve compliance in a health survey conducted by telephone. Public Health Reports, 105, 524-529. 
Howard, D.J. (1990). The influence of verbal responses to common greetings on compliance behavior: The foot-in-the-mouth effect. Journal of Applied Social Psychology, 20, 1185-1196.

*Joule R.V. (1987). Tobacco deprivation: The foot-in-the-door technique versus the low-ball technique. European Journal of Social Psychology, 17, 361-365.

*Joule, R.V., Fointiat, V., Pasquier, C. \& Mugny, G. (1991). Behavioral conversion in a compliance paradigm : a replication and refinement. European Journal of Social Psychology, 21, 365-369.

*Katzev, R. \& Brownstein, R. (1989). The influence of enlightenment on compliance. The Journal of Social Psychology, 129, 335-347.

Kiesler, C. A. (1971). The psychology of commitment. Experiments linking behavior to belief. New York: Academic Press.

Kilbourne, B. (1989). A cross-cultural investigation of foot-in-the-door compliance induction procedure. Journal of Cross-Cultural Psychology, 20, 3-38.

*Maj, K. (2002). Zachowanie uprzejme jako determinanta skuteczności techniki wpływu społecznego 'niska piłka' [Polite behavior as a condition determining the effectiveness of social influence called 'the low-ball]. Studia Psychologiczne, 40(3), 93-109.

*Masli, M., \& Terveen, L. (2012). Evaluating compliance-without-pressure techniques for increasing participation in online communities. In Proceedings of the 2012 ACM annual conference on Human Factors in Computing Systems, ACM (2012), 2915-2924.

*Motes, W. \& Woodside, A. (1979). Influence of low-balling on buyers' compliance. Journal of Psychology, 101, 219-221.

*Motes, W.H., Brown, R.E., Ezell, H.F. \& Hudson, G.I. (1986). The Influence of "LowBalling" on Buyers' Compliance: Revisited. Psychology \& Marketing, 3(2), 79-86. 
O'Keefe, D.J. \& Hale, S.L. (2001). An odds-ratio-based meta-analysis of research on the door-in-the-face influence strategy. Communication Reports, 14, 31-38.

Pascual, A. \& Guéguen, N. (2005). Foot-in-the-door and door-in-the-face: a comparative meta-analytic study. Psychological Reports, 96, 122-128.

Pascual A. \& Guéguen N. (2004). Cultural differences in altruistic behavior: A quasireplication of Uranowitz' "Foot-in-the-door with implicit demand". Psychological Reports, 94, 767-770.

Pascual, A., Oteme, C., Samson, L., Wang, Q., Halimi-Falkowicz, S., Souchet, L., Girandola, F., Guéguen, N. \& Joule, R.V. (2012). Cross cultural investigation of compliance without pressure: the "you are free to .. " technique in France, Ivory Coast, Romania, Russia and China. Cross-Cultural Research: The Journal of Comparative Social Science, 46, 394-416

Pratkanis, A. R. (2007). The science of social influence. New York: Psychological Press.

Wang, T., Brownstein, R., \& Katzev, R. (1989). Promoting charitable behaviour with compliance techniques. Applied Psychology: An International Review, 38, 165-183.

Weyant, J. M. (1996). Application of compliance techniques to Direct-Mail requests for charitable donations. Psychology \& Marketing, 13, 157-170. 
Table 1

Articles, Compliance Effects and Sample-Sizes

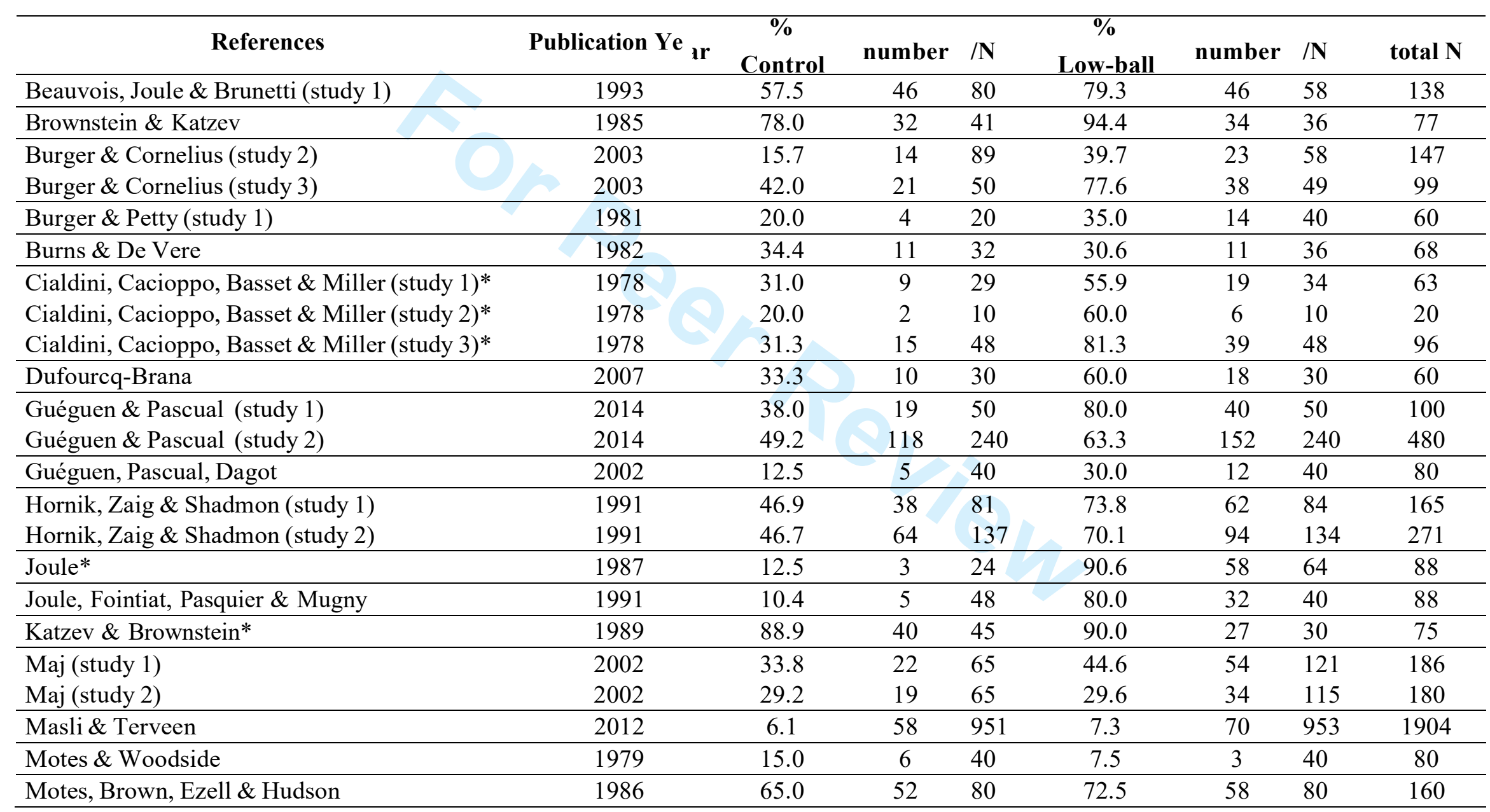

\footnotetext{
* These studies measured both verbal and behavioral compliance; verbal compliance rates are reported here.
} 
Table 2

Overall Effect-Sizes and Explained Variance across Moderators Associated with Frequency of Compliance

\begin{tabular}{|c|c|c|c|c|c|c|c|}
\hline & $\mathbf{k}$ & $\mathbf{N}^{*}$ & $\mathbf{r}$ & OR & $\begin{array}{c}\% \\
\text { explained }\end{array}$ & Lower $80 \%$ & Upper $80 \%$ \\
\hline Full Sample & 45 & $\overline{5218}$ & 0.16 & 2.47 & 27 & -0.04 & 0.38 \\
\hline Without Internet & 44 & 3314 & 0.23 & 3.72 & 37 & 0.05 & 0.41 \\
\hline \multicolumn{8}{|l|}{ Nature of Request } \\
\hline Business & 8 & 1042 & 0.13 & 1.68 & 51 & 0.02 & 0.23 \\
\hline Self-Interested & 7 & 410 & 0.47 & 25.24 & 32 & 0.28 & 0.67 \\
\hline Prosocial & 11 & 611 & 0.25 & 3.62 & 100 & & \\
\hline Other & 19 & 3155 & 0.11 & 1.93 & 29 & -0.05 & 0.26 \\
\hline Other w/o Int & 18 & 1251 & 0.23 & 3.92 & 50 & 0.08 & 0.38 \\
\hline \multicolumn{8}{|l|}{ Cost to 1 arget } \\
\hline Low & 5 & 409 & 0.15 & 3.07 & 54 & 0.02 & 0.28 \\
\hline Moderate & 23 & 3742 & 0.10 & 1.70 & 33 & -0.04 & 0.24 \\
\hline Moderate w/o Int & 22 & 1838 & 0.18 & 2.41 & 45 & 0.03 & 0.33 \\
\hline High & 17 & 1067 & 0.35 & 8.50 & 44 & 0.19 & 0.51 \\
\hline \multicolumn{8}{|l|}{ Country } \\
\hline France & 20 & 1218 & 0.34 & 9.64 & 40 & 0.16 & 0.52 \\
\hline United States & 21 & 3198 & 0.09 & 1.56 & 39 & -0.05 & 0.21 \\
\hline United States w/o Int & 20 & 1294 & 0.17 & 2.25 & 52 & 0.02 & 0.32 \\
\hline \multicolumn{8}{|l|}{ I 1me to Kesponse } \\
\hline Deferred & 13 & 1235 & 0.31 & 5.74 & 29 & 0.12 & 0.50 \\
\hline Immediate & 32 & 3983 & 0.11 & 1.90 & 38 & -0.04 & 0.25 \\
\hline Immediate w/o Int & 31 & 2079 & 0.18 & 2.88 & 50 & 0.03 & 0.34 \\
\hline \multicolumn{8}{|l|}{ Size of Target } \\
\hline Group & 3 & 397 & 0.07 & 1.41 & 100 & & \\
\hline Individual & 42 & 4821 & 0.16 & 2.59 & 26 & -0.03 & 0.36 \\
\hline
\end{tabular}




\begin{tabular}{|c|c|c|c|c|c|c|c|}
\hline Individual w/o Int & 41 & 2917 & 0.25 & 4.25 & 39 & 0.07 & 0.43 \\
\hline \multicolumn{8}{|l|}{ Iviode } \\
\hline Face to face & 31 & 2159 & 0.18 & 2.84 & 52 & 0.04 & 0.33 \\
\hline Phone & 13 & 1155 & 0.32 & 6.17 & 29 & 0.13 & 0.51 \\
\hline Internet & 1 & 1904 & 0.03 & 1.21 & & & \\
\hline \multicolumn{8}{|l|}{ Ivieasure } \\
\hline Behavioral & 33 & 4148 & 0.14 & 2.28 & 30 & -0.03 & 0.31 \\
\hline Behavioral w/o Int & 32 & 2244 & 0.24 & 3.90 & 48 & 0.08 & 0.39 \\
\hline Verbal & 12 & 1070 & 0.22 & 3.38 & 23 & -0.02 & 0.43 \\
\hline \multicolumn{8}{|l|}{ Sex of Experimenter } \\
\hline Female & 5 & 432 & 0.28 & 3.58 & 100 & & \\
\hline Male & 15 & 1184 & 0.16 & 2.57 & 100 & & \\
\hline Mixed & 25 & 3602 & 0.14 & 2.34 & 18 & -0.09 & 0.37 \\
\hline Mixed w/o Int & 24 & 1698 & 0.27 & 4.88 & 25 & 0.02 & 0.52 \\
\hline \multicolumn{8}{|l|}{ Sex of Subjects } \\
\hline Female & 7 & 400 & 0.14 & 2.54 & 78 & 0.05 & 0.23 \\
\hline Male & 9 & 468 & 0.32 & 8.13 & 62 & 0.19 & 0.44 \\
\hline Mixed & 29 & 4350 & 0.14 & 2.17 & 23 & -0.05 & 0.33 \\
\hline Mixed w/o Int & 28 & 2446 & 0.23 & 3.41 & 32 & 0.04 & 0.42 \\
\hline Mixed w/o Int FtF only & 15 & 1291 & 0.15 & 2.01 & 58 & 0.03 & 0.26 \\
\hline \multicolumn{8}{|l|}{ Country X Nature } \\
\hline \multicolumn{8}{|l|}{ France } \\
\hline Prosocial & 4 & 180 & 0.31 & 5.00 & 100 & & \\
\hline Self-Interested & 7 & 410 & 0.47 & 25.24 & 32 & 0.28 & 0.67 \\
\hline Other & 9 & 628 & 0.26 & 6.20 & 63 & 0.15 & 0.37 \\
\hline \multicolumn{8}{|l|}{ United States } \\
\hline Business & 4 & 240 & 0.02 & 0.98 & 100 & & \\
\hline Prosocial & 7 & 431 & 0.22 & 3.16 & 91 & 0.18 & 0.27 \\
\hline Other & 10 & 2527 & 0.07 & 1.44 & 30 & -0.06 & 0.19 \\
\hline Other w/o Int & 9 & 623 & 0.20 & 2.46 & 44 & 0.03 & 0.36 \\
\hline
\end{tabular}




\section{Personality and Social Psychology Review}

* $\mathrm{N}$ full sample is higher than table 1 because some studies had verbal and behavioral compliance rates. 